\title{
Self-Assembly of 1:2 Inclusion Complexes between a Metallocycle Host and Dihydroxyaromatic Guests: A Redox Controlled Complexation Process
}

\author{
Víctor Blanco, Marcos Chas, Dolores Abella, Elena Pía, Carlos Platas-Iglesias, Carlos Peinador* \\ and José M. Quintela*. \\ Departamento de Química Fundamental, Facultad de Ciencias, Universidade da Coruña. Campus A Zapateira, \\ 15071, A Coruña, Spain. Fax: 3481 167065; Tel: 3481167000. \\ E-mail: capeveqo@udc.es (C.P.); jqqoqf@udc.es (J.M.Q.)
}

\section{Supporting Information}

\section{Table of Contents}

General Methods

$\mathrm{S} 2$

Synthesis of Compounds $\mathbf{1} \cdot 2 \mathrm{NO}_{3}, \mathbf{3} \cdot 8 \mathrm{NO}_{3},(\mathrm{HQ})_{2} \subset \mathbf{3} \cdot 8 \mathrm{NO}_{3}$, and $(\mathrm{DHNP})_{2} \subset \mathbf{3} \cdot 8 \mathrm{NO}_{3}$

NMR data of $1 \cdot 2 \mathrm{NO}_{3}$

S4-S6

NMR data of $\mathbf{3} \cdot 8 \mathrm{NO}_{3}$

S7-S9

DOSY Experiment of $\mathbf{3} \cdot 8 \mathrm{NO}_{3}$

$\mathrm{S} 10$

NMR data of $(\mathrm{HQ})_{2} \subset \mathbf{3} \cdot 8 \mathrm{NO}_{3}$

$$
\text { S11-S13 }
$$

NMR data of $(\text { DHNP })_{2} \subset \mathbf{3} \cdot 8 \mathrm{NO}_{3}$

${ }^{1} \mathrm{H}$ NMR spectra of oxidation/reduction of complexes 


\section{General Methods}

Compound $1 \cdot 2 \mathrm{Br}^{1}$ was prepared according to literature procedure. All reagents used were commercial grade chemicals from freshly opened containers. Milli-Q water was purified with a Millipore Gradient A10 apparatus. Proton and carbon nuclear magnetic resonance spectra were recorded on a Bruker AC200F or Bruker Avance 500, using the deuterated solvent as lock and the residual protiated solvent as internal standard. DOSY experiments were referenced using the value $1.92 \times 10^{-9} \mathrm{~m}^{2} / \mathrm{s}$ for the DHO signal in $\mathrm{D}_{2} \mathrm{O}$ at $298 \mathrm{~K}^{2}$. Mass spectrometry experiments were carried out in a LC-Q-q-TOF Applied Biosystems QSTAR Elite spectrometer. UV-Vis spectra were obtained using a Perkin Elmer Lambda 900 spectrometer. Cyclic voltammograms were measured using Autolab equipment with a PGSTAT20 potentiostat at room temperature. All measurements were made by using a three-electrode system in deareated solvents containing $\mathrm{NaNO}_{3}(0.1 \mathrm{M})$ as a supporting electrolyte: a glassy-carbon electrode as a working electrode (diameter $2 \mathrm{~mm}$ ), a glassycarbon wire as a counter-electrode, and $\mathrm{Ag} / \mathrm{Ag}^{+}$as a reference electrode. Melting points were measured using Stuart Scientific SMP3 apparatus. Microanalyses for C, H and N were performed by the elemental analyses general service of the University of A Coruña.

Crystal Structure Analysis: The structures were solved by direct methods and refined with the full-matrix least-squares procedure (SHELX-97) ${ }^{3}$ against $F^{2}$. The X-ray diffraction data were collected on a Bruker X8 APEXII or a Bruker SMART 1k diffractometer. Hydrogen atoms were placed in idealized positions with $U_{\mathrm{eg}}(\mathrm{H}) 1.2 U_{\mathrm{eg}}(\mathrm{C})$ and were allowed to ride on their parent atoms.

\section{Ligand $1 \cdot 2 \mathrm{NO}_{3}$}

Ligand 1.2Br $(0.80 \mathrm{~g}, 1.64 \mathrm{mmol})$ was dissolved in water $(50 \mathrm{~mL})$ and stirred with $\mathrm{AgNO}_{3}(0.60 \mathrm{~g}$, $3.28 \mathrm{mmol}$ ) in the absence of light for $18 \mathrm{~h}$ at room temperature. The suspension was then filtered, and the filtrate concentrated in vacuo to give $1 \cdot 2 \mathrm{NO}_{3}(0.72,96 \%)$ as a light brown solid; $\mathrm{mp}\left({ }^{\circ} \mathrm{C}\right)$ : 229-233 (decomp); $\left.{ }^{1} \mathrm{H} \mathrm{NMR} \mathrm{(500} \mathrm{MHz,} \mathrm{D}_{2} \mathrm{O}\right) \delta: 7.47(2 \mathrm{H}, \mathrm{s}) ; 7.82(4 \mathrm{H}, \mathrm{d}, J=6.4 \mathrm{~Hz}) ; 8.61(4 \mathrm{H}, \mathrm{d}$, $J=7.0 \mathrm{~Hz}) ; 8.74(4 \mathrm{H}, \mathrm{d}, J=6.3 \mathrm{~Hz}) ; 9.41(4 \mathrm{H}, \mathrm{d}, J=6.9 \mathrm{~Hz}) ;{ }^{13} \mathrm{CNMR}\left(125 \mathrm{MHz}, D_{2} \mathrm{O}\right) \delta: 77.3$ $\left(\mathrm{CH}_{2}\right) ; 122.6(\mathrm{CH}) ; 127.1(\mathrm{CH}) ; 141.6(\mathrm{C}) ; 145.5(\mathrm{CH}) ; 150.0(\mathrm{CH}) ; 157.8(\mathrm{C}) . \operatorname{EM}-E S(\mathrm{~m} / \mathrm{z}): 325.1$ $\left[\mathrm{M}-2 \mathrm{NO}_{3}{ }^{-}\right]^{+} ; 163.1\left[\mathrm{M}-2 \mathrm{NO}_{3}{ }^{-}-\mathrm{H}^{+}\right]^{2+}$. Anal. Calcd. $\mathrm{C}_{21} \mathrm{H}_{18} \mathrm{~N}_{6} \mathrm{O}_{6}: \mathrm{C}, 56.00 ; \mathrm{H}, 4.03, \mathrm{~N}, 18.66 \%$. Found. C, 55.83; H, 4.07; N, 18.39 .

\section{Metallocycle 3·8 $\mathrm{NO}_{3}$}

To a solution of ligand $\mathbf{1} \cdot 2 \mathrm{NO}_{3}(18.0 \mathrm{mg} ; 0.040 \mathrm{mmol})$ in $\mathrm{D}_{2} \mathrm{O}(4.0 \mathrm{~mL})$ palladium complex $2(11.6$ $\mathrm{mg}, 0.040 \mathrm{mmol})$ was added. ${ }^{1} \mathrm{H} N M R\left(500 \mathrm{MHz}, \mathrm{D}_{2} \mathrm{O}\right) \delta: 2.92(8 \mathrm{H}, \mathrm{s}) ; 7.48(4 \mathrm{H}, \mathrm{s}) 8.00(8 \mathrm{H}, \mathrm{d}, J=$ $6.8 \mathrm{~Hz}) ; 8.51(8 \mathrm{H}, \mathrm{d}, J=6.8 \mathrm{~Hz}) ; 8.95(8 \mathrm{H}, \mathrm{d}, J=6.7 \mathrm{~Hz}) ; 9.40(8 \mathrm{H}, \mathrm{d}, J=6.8 \mathrm{~Hz}) ;{ }^{13} C N M R(125$ $\left.M H z, D_{2} \mathrm{O}\right) \delta: 46.8\left(\mathrm{CH}_{2}\right) ; 77.4\left(\mathrm{CH}_{2}\right) ; 125.2(\mathrm{CH}) ; 127.4(\mathrm{CH}) ; 144.2(\mathrm{C}) ; 145.8(\mathrm{CH}) ; 152.2(\mathrm{CH})$; $154.9(\mathrm{C})$.

\section{Inclusion Complex $(\mathrm{HQ})_{2} \subset \mathbf{3} \cdot 8 \mathrm{NO}_{3}$}

To a solution of ligand $1 \cdot 2 \mathrm{NO}_{3}(9.0 \mathrm{mg}, 0.020 \mathrm{mmol})$ in $\mathrm{D}_{2} \mathrm{O}(2.0 \mathrm{~mL})$ were added the palladium complex $2(5.8 \mathrm{mg}, 0.020 \mathrm{mmol})$ and HQ $(2.2 \mathrm{mg}, 0.020 \mathrm{mmol}) .{ }^{1} \mathrm{H} \mathrm{NMR}\left(500 \mathrm{MHz}, \mathrm{D}_{2} \mathrm{O}\right) \delta: 3.01$ $(8 \mathrm{H}, \mathrm{s}) ; 6.81(8 \mathrm{H}, \mathrm{s}) ; 7.54(4 \mathrm{H}, \mathrm{s}) 8.05(8 \mathrm{H}, \mathrm{d}, J=7.0 \mathrm{~Hz}) ; 8.57(8 \mathrm{H}, \mathrm{d}, J=6.9 \mathrm{~Hz}) ; 9.02(8 \mathrm{H}, \mathrm{d}, J=$ $6.9 \mathrm{~Hz}) ; 9.46(8 \mathrm{H}, \mathrm{d}, J=7.0 \mathrm{~Hz}) .{ }^{13} \mathrm{C} N M R\left(125 \mathrm{MHz}, \mathrm{D}_{2} \mathrm{O}\right) \delta: 46.9\left(\mathrm{CH}_{2}\right) ; 77.0\left(\mathrm{CH}_{2}\right) ; 116.4(\mathrm{CH})$; $125.3(\mathrm{CH}) ; 127.4(\mathrm{CH}) ; 144.2(\mathrm{C}) ; 145.8(\mathrm{CH}) ; 149.0(\mathrm{C}) ; 152.3(\mathrm{CH}) ; 154.9(\mathrm{C})$.

\footnotetext{
${ }^{1}$ Foucher, D. A.; Macartney, D. H.; Warrack, L. J.; Wilson, J. P. Inorg. Chem. 1993, 32, 3425-3432.

2 L. G: Longsworth, Phys. Chem. 1960, 1914-1917.

${ }^{3}$ G. M Sheldrick, SHELX-97, An Integrated System for Solving and Refining Crystal Structures from Diffraction Data, University of Göttingen, Germany, 1997.
} 
Single red crystals of $(\mathrm{HQ})_{2} \subset \mathbf{3} \cdot 8 \mathrm{NO}_{3}$ suitable for X-ray crystallography were grown from $3 \mathrm{M}$ $\mathrm{NaNO}_{3}$ solution of $\mathbf{1} \cdot 2 \mathrm{NO}_{3}, \mathbf{2}$, and HQ ( in a 1:1:5 mol ratio).

\section{Inclusion Complex (DHNP $)_{2} \subset \mathbf{3} \cdot 8 \mathrm{NO}_{3}$}

To a solution of ligand $\mathbf{1} \cdot 2 \mathrm{NO}_{3}(9.0 \mathrm{mg}, 0.020 \mathrm{mmol})$ in $\mathrm{D}_{2} \mathrm{O}(2.0 \mathrm{~mL})$ were added the palladium complex 2 (5.8 mg, $0.020 \mathrm{mmol})$ and 1,5-dihydroxynaphthalene $(3.2 \mathrm{mg}, 0.020 \mathrm{mmol})$. The solution was stirred for $24 \mathrm{~h}$ at room temperature. ${ }^{l} \mathrm{H} \mathrm{NMR}\left(500 \mathrm{MHz}, \mathrm{D}_{2} \mathrm{O}\right) \delta: 2.97(8 \mathrm{H}, \mathrm{s}) ; 5.01$ $(4 \mathrm{H}, \mathrm{d}, J=7.5 \mathrm{~Hz}) ; 6.00(4 \mathrm{H}, \mathrm{d}, J=7.5 \mathrm{~Hz}) ; 6.19(4 \mathrm{H}, \mathrm{t}, J=7.7 \mathrm{~Hz}) ; 7.45(4 \mathrm{H}, \mathrm{s}) 7.97(8 \mathrm{H}, \mathrm{d}, J=$ $6.2 \mathrm{~Hz}) ; 8.41(8 \mathrm{H}, \mathrm{d}, J=6.3 \mathrm{~Hz}) ; 9.07(8 \mathrm{H}, \mathrm{d}, J=6.8 \mathrm{~Hz}) ; 9.39(8 \mathrm{H}, \mathrm{d}, J=6.9 \mathrm{~Hz}) .{ }^{13} C N M R(125$ $\left.\mathrm{MHz}, \mathrm{D}_{2} \mathrm{O}\right) \delta: 46.8\left(\mathrm{CH}_{2}\right) ; 77.0\left(\mathrm{CH}_{2}\right)^{*} ; 107.9(\mathrm{CH}) ; 111.4(\mathrm{CH}) ; 124.0(\mathrm{C}) ; 124.2(\mathrm{CH}) ; 125.1$ $(\mathrm{CH}) ; 127.1(\mathrm{CH}) ; 143.4(\mathrm{C}) ; 145.4(\mathrm{CH}) ; 150.3(\mathrm{C}) ; 152.3(\mathrm{CH}) ; 154.2(\mathrm{C})$.

Single violet crystals of (DHNP) $)_{2} \subset \mathbf{3} \cdot 8 \mathrm{NO}_{3}$ suitable for X-ray crystallography were grown from 30 $\mathrm{mM} \mathrm{NaNO} 3$ solution of $\mathbf{1} \cdot 2 \mathrm{NO}_{3}, \mathbf{2}$, and DHNP ( in a 1:1:1 mol ratio). 


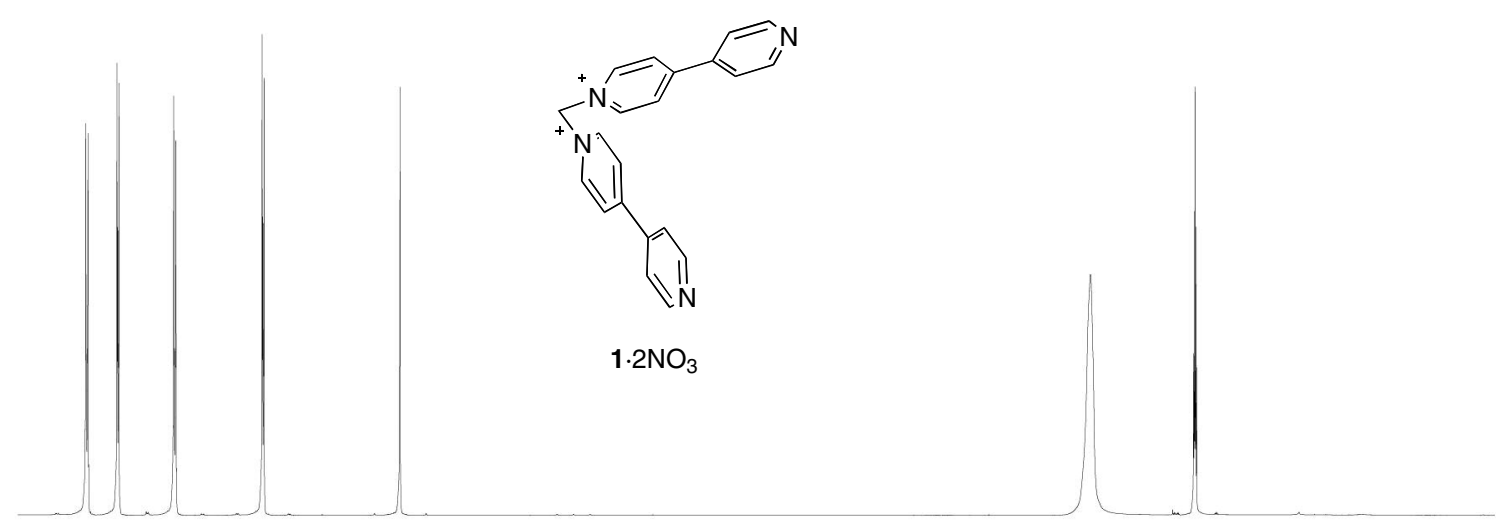

\begin{tabular}{c|c|c|c|c|c|c|c|c|c|c|c|c|}
\hline \\
$\operatorname{ppm}(\mathrm{t} 1)$
\end{tabular}
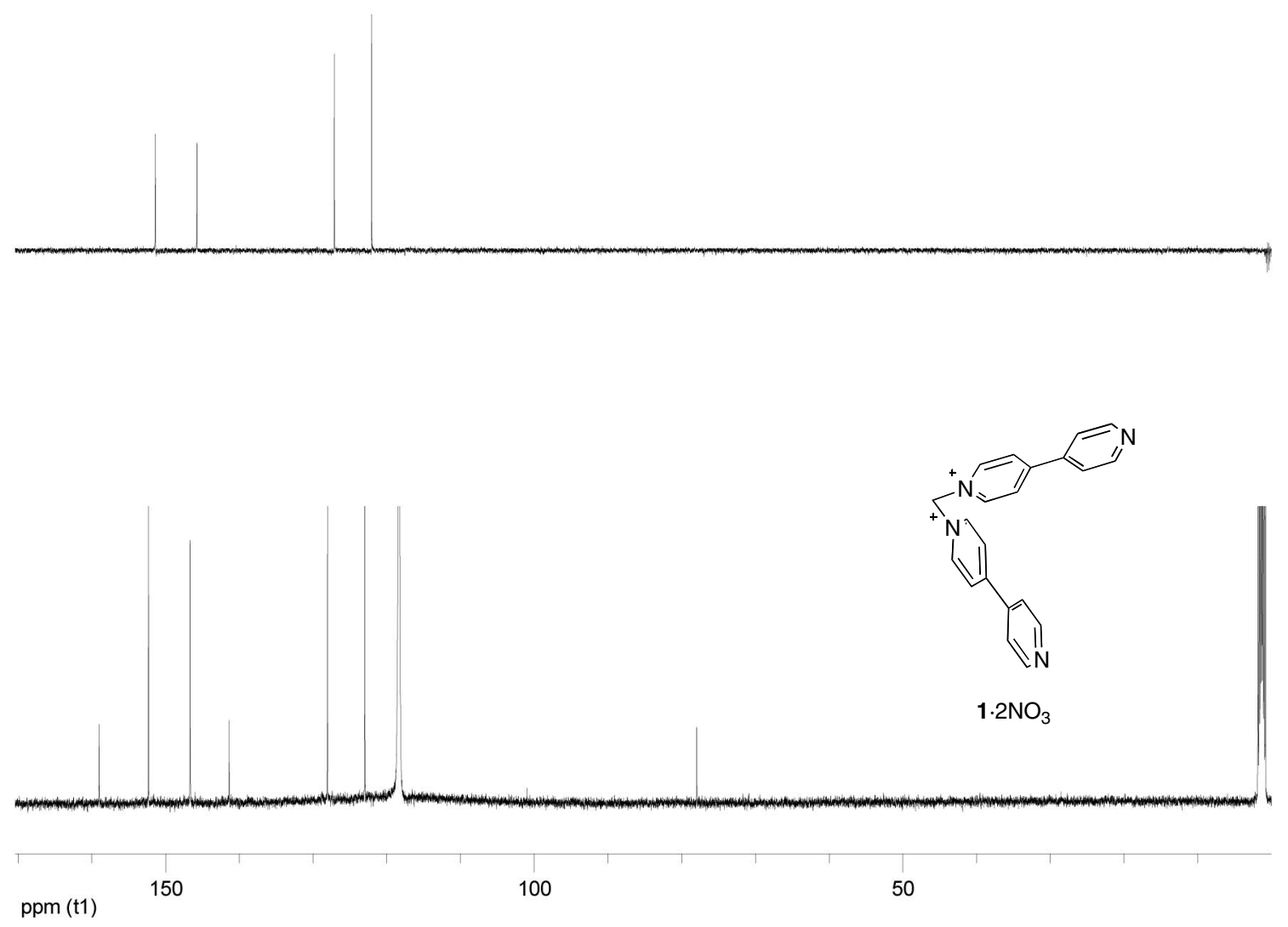

Figure $\mathrm{S1}{ }^{1} \mathrm{H}$ NMR $\left(500 \mathrm{MHz}, \mathrm{D}_{2} \mathrm{O}\right)$ (top) and ${ }^{13} \mathrm{C}$ NMR $\left(125 \mathrm{MHz}, \mathrm{D}_{2} \mathrm{O}\right)$ (bottom) spectra of $1.2 \mathrm{NO}_{3}$ 

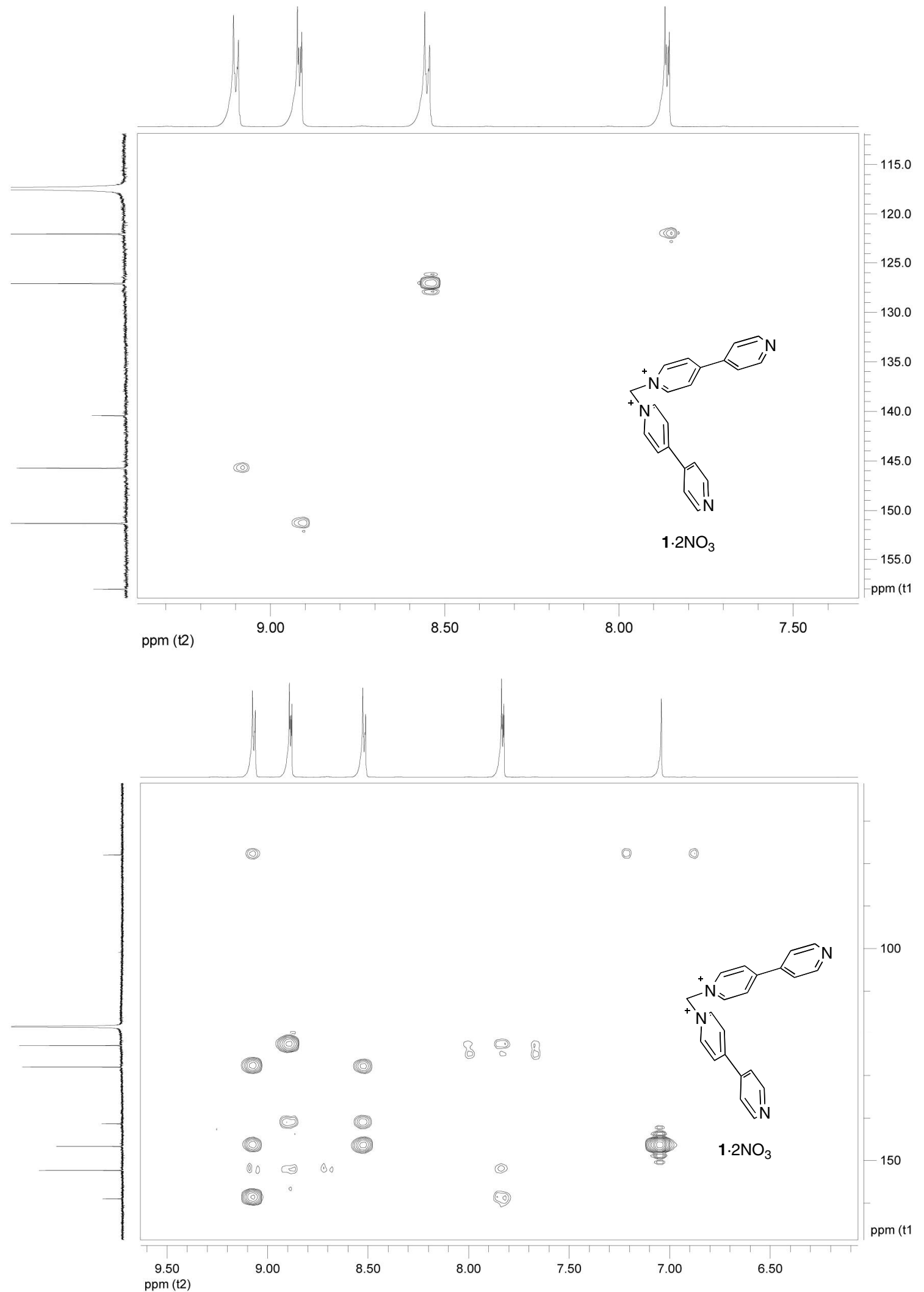

Figure S2 HSQC (top) and HMBC (500 MHz, $\mathrm{D}_{2} \mathrm{O}$ ) (bottom) spectra of $\mathbf{1} \cdot 2 \mathrm{NO}_{3}$. 


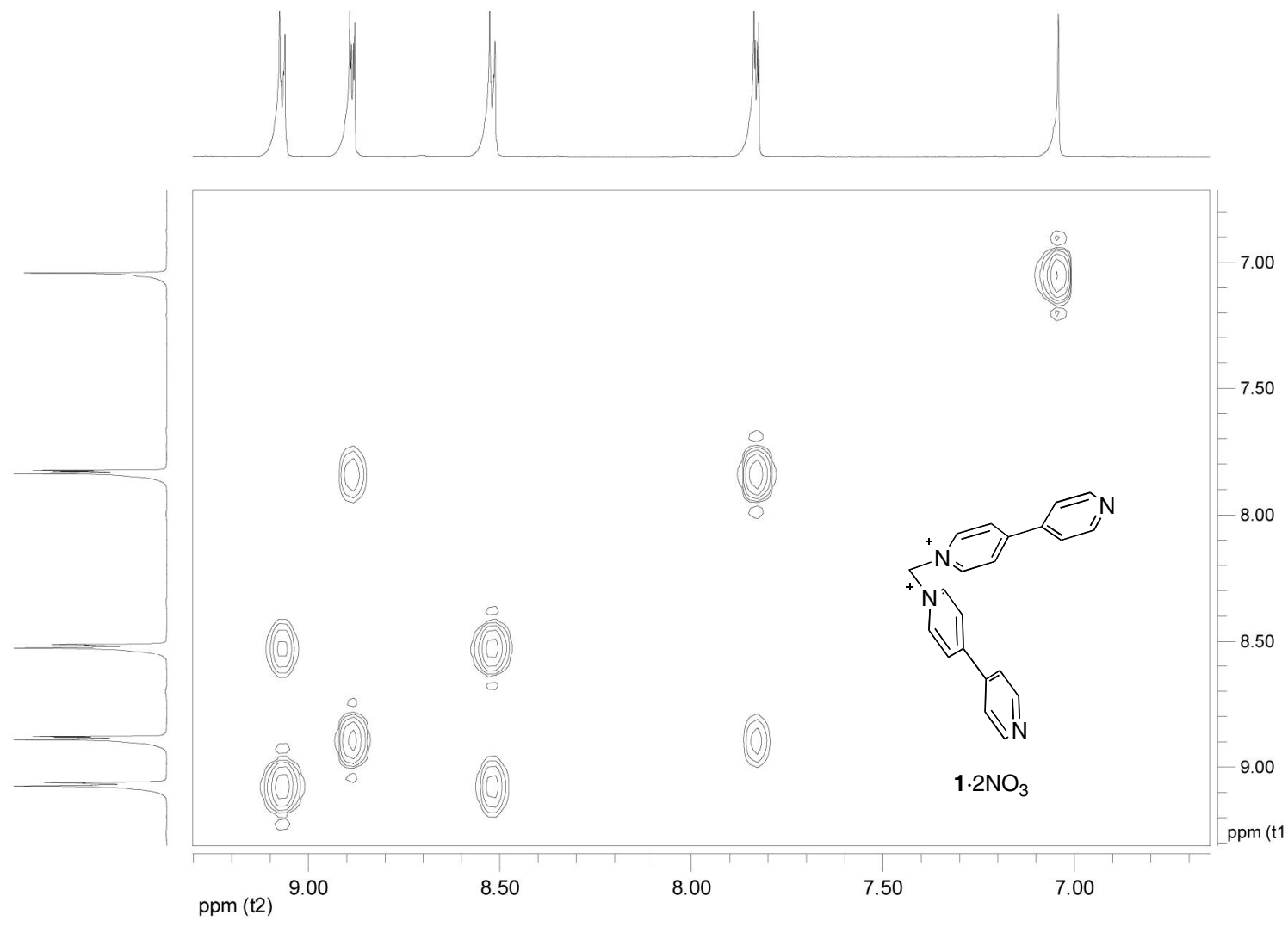

Figure S3 COSY $\left(500 \mathrm{MHz}, \mathrm{D}_{2} \mathrm{O}\right)$ spectrum of $\mathbf{1} \cdot 2 \mathrm{NO}_{3}$. 


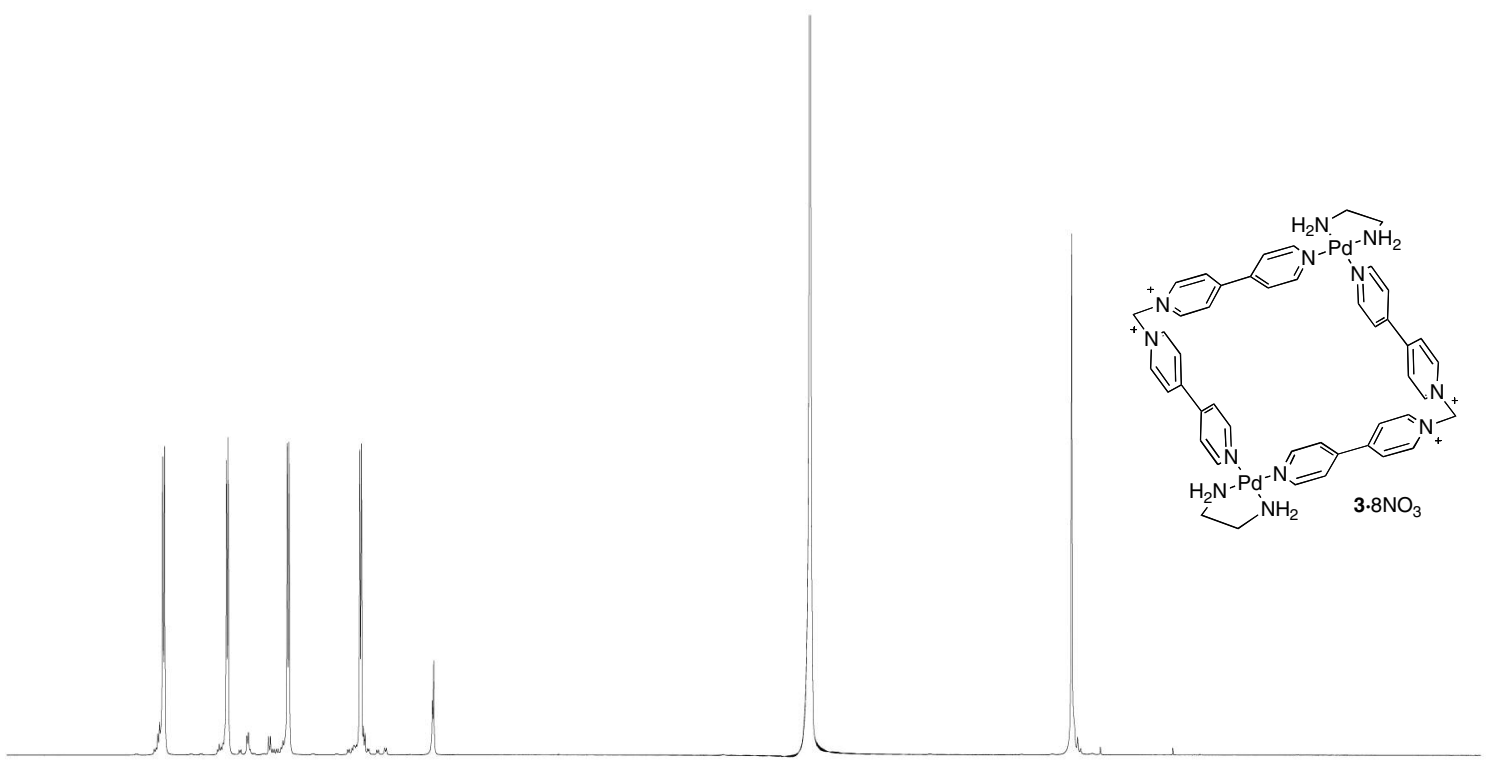

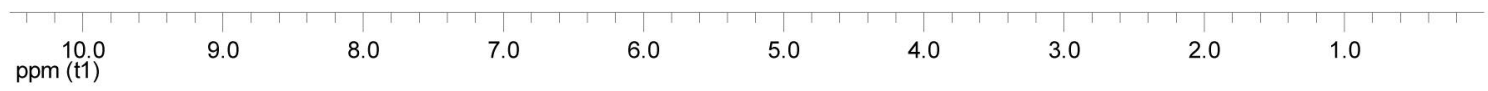
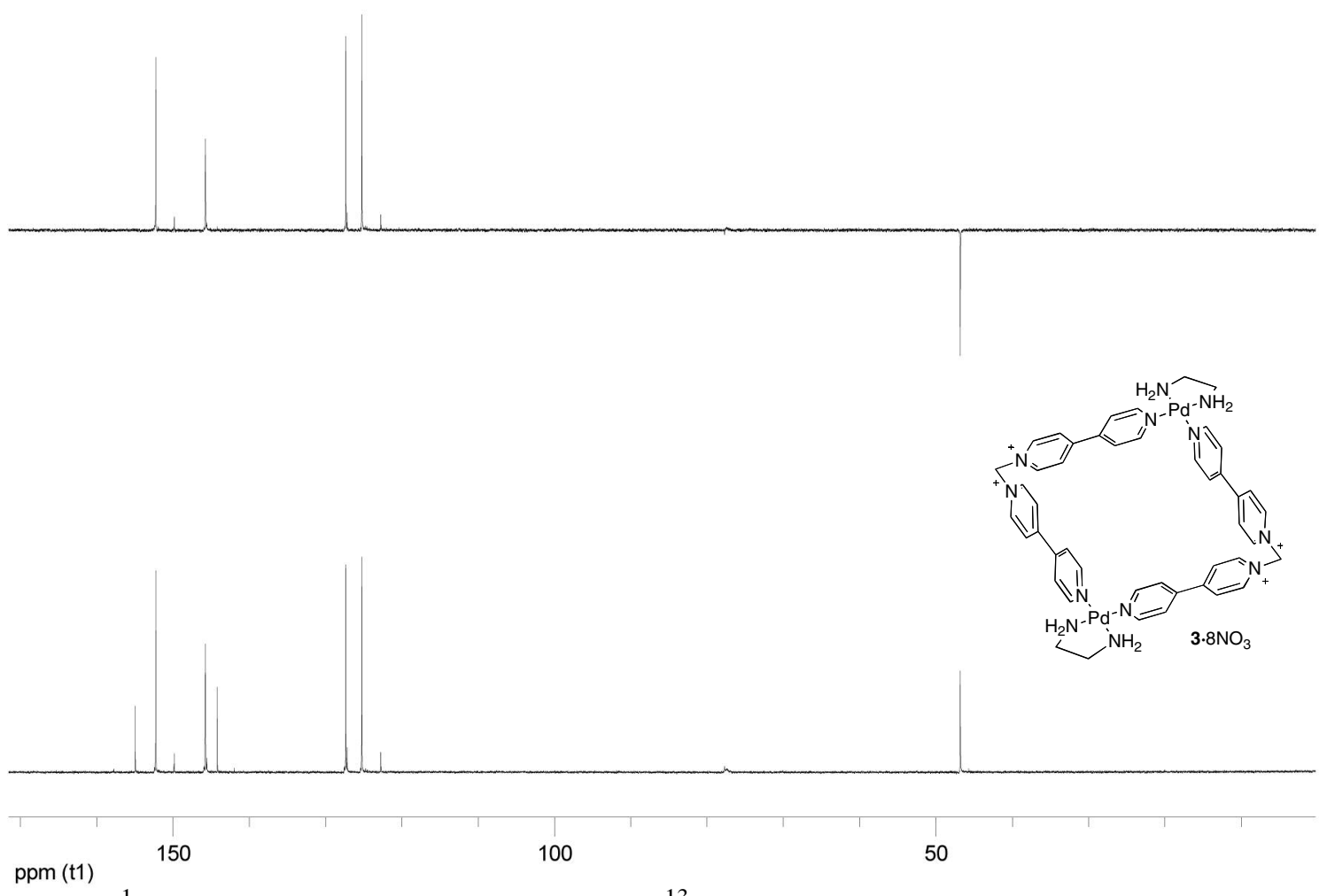

Figure S4 ${ }^{1} \mathrm{H}$ NMR (500 MHz, $\mathrm{D}_{2} \mathrm{O}$ ) (top) and ${ }^{13} \mathrm{C}$ NMR $\left(125 \mathrm{MHz}, \mathrm{D}_{2} \mathrm{O}\right)$ (bottom) spectra of 3. $8 \mathrm{NO}_{3}$. 

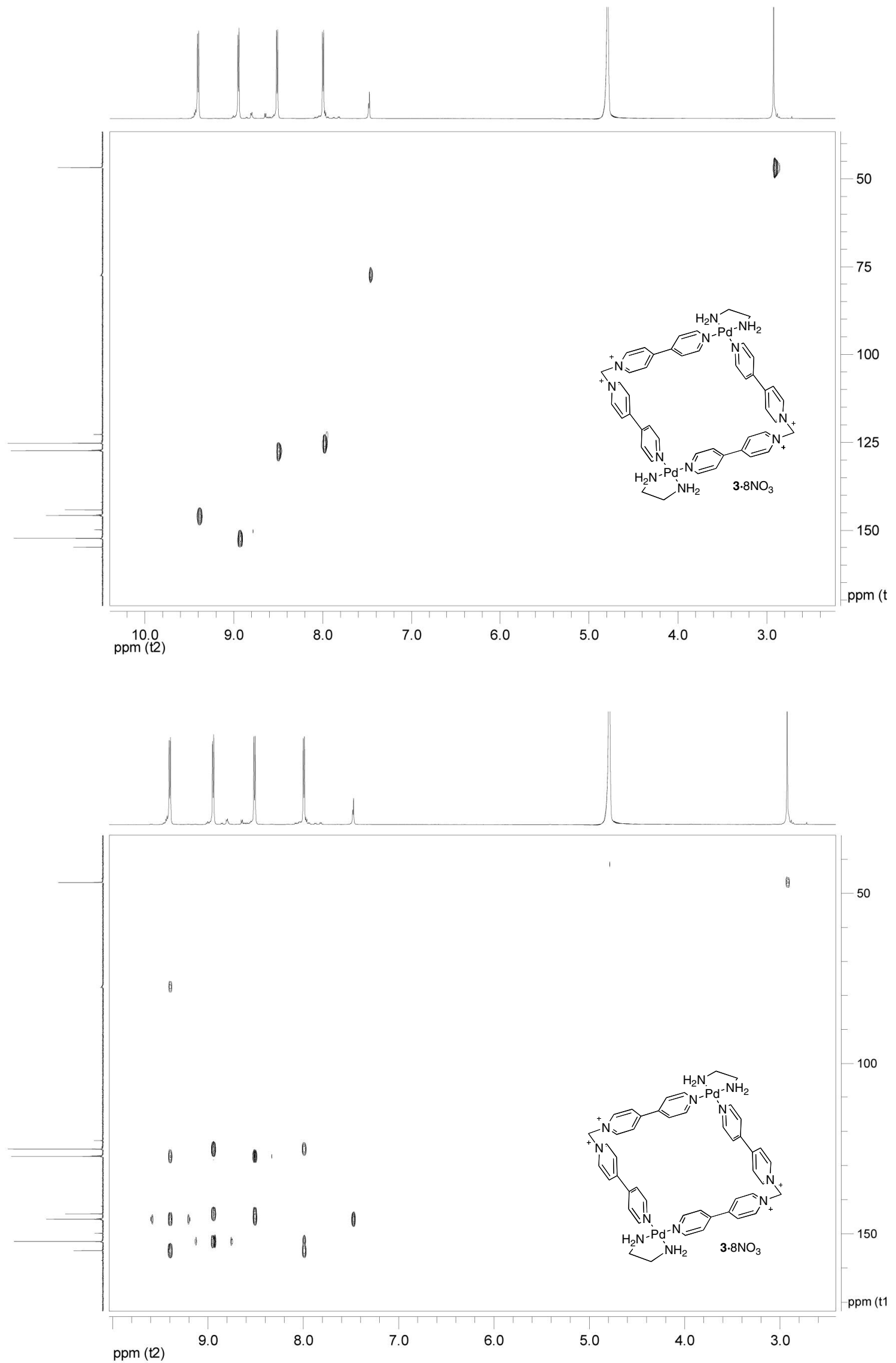

Figure S5 HSQC (500 MHz, $\mathrm{D}_{2} \mathrm{O}$ ) (top) and $\mathrm{HMBC}\left(500 \mathrm{MHz}, \mathrm{D}_{2} \mathrm{O}\right.$ ) (bottom) spectra of 3.8 $\mathrm{NO}_{3}$. 


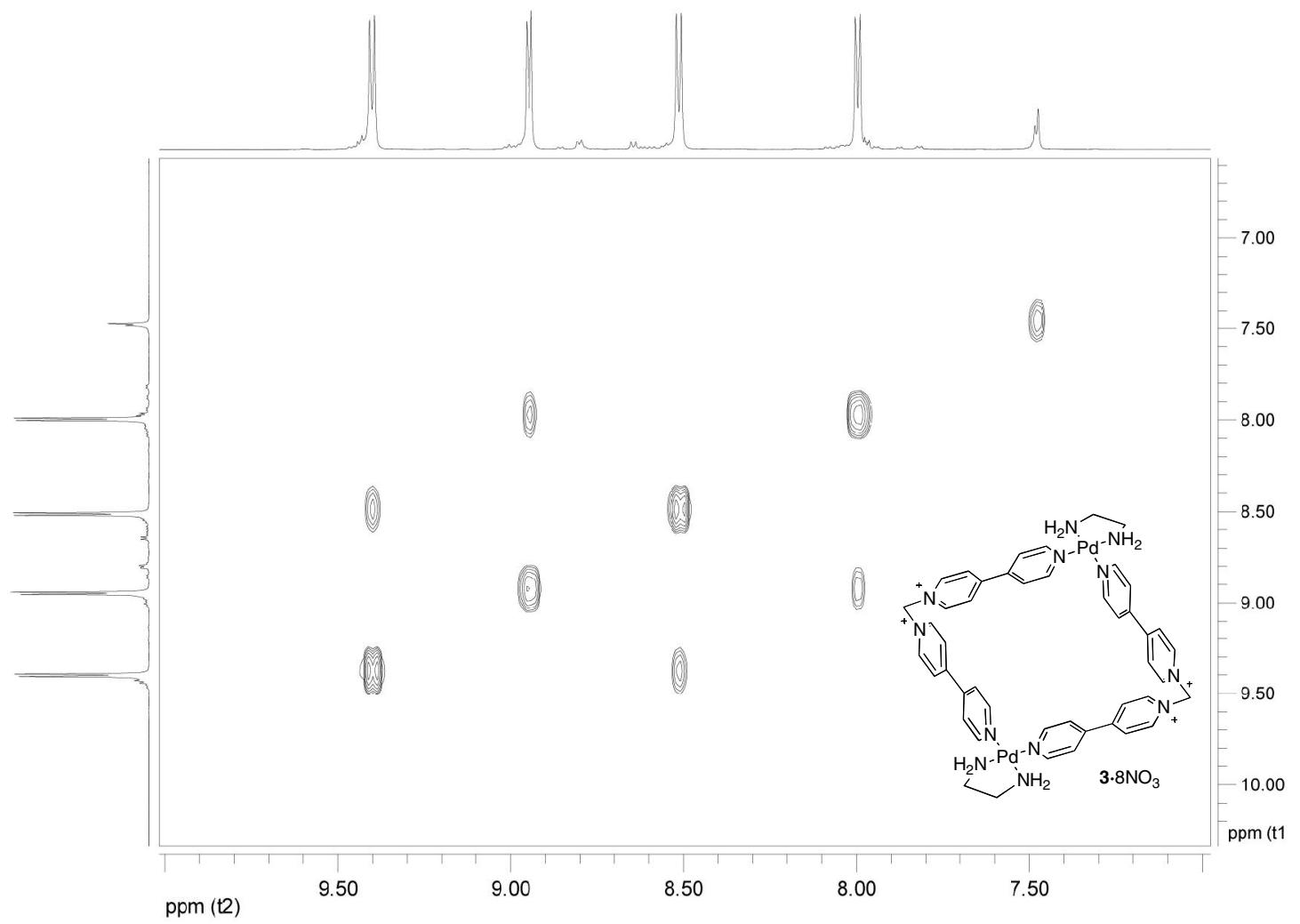

Figure S6 COSY $\left(500 \mathrm{MHz}, \mathrm{D}_{2} \mathrm{O}\right)$ spectrum of $\mathbf{3} \cdot 8 \mathrm{NO}_{3}$.

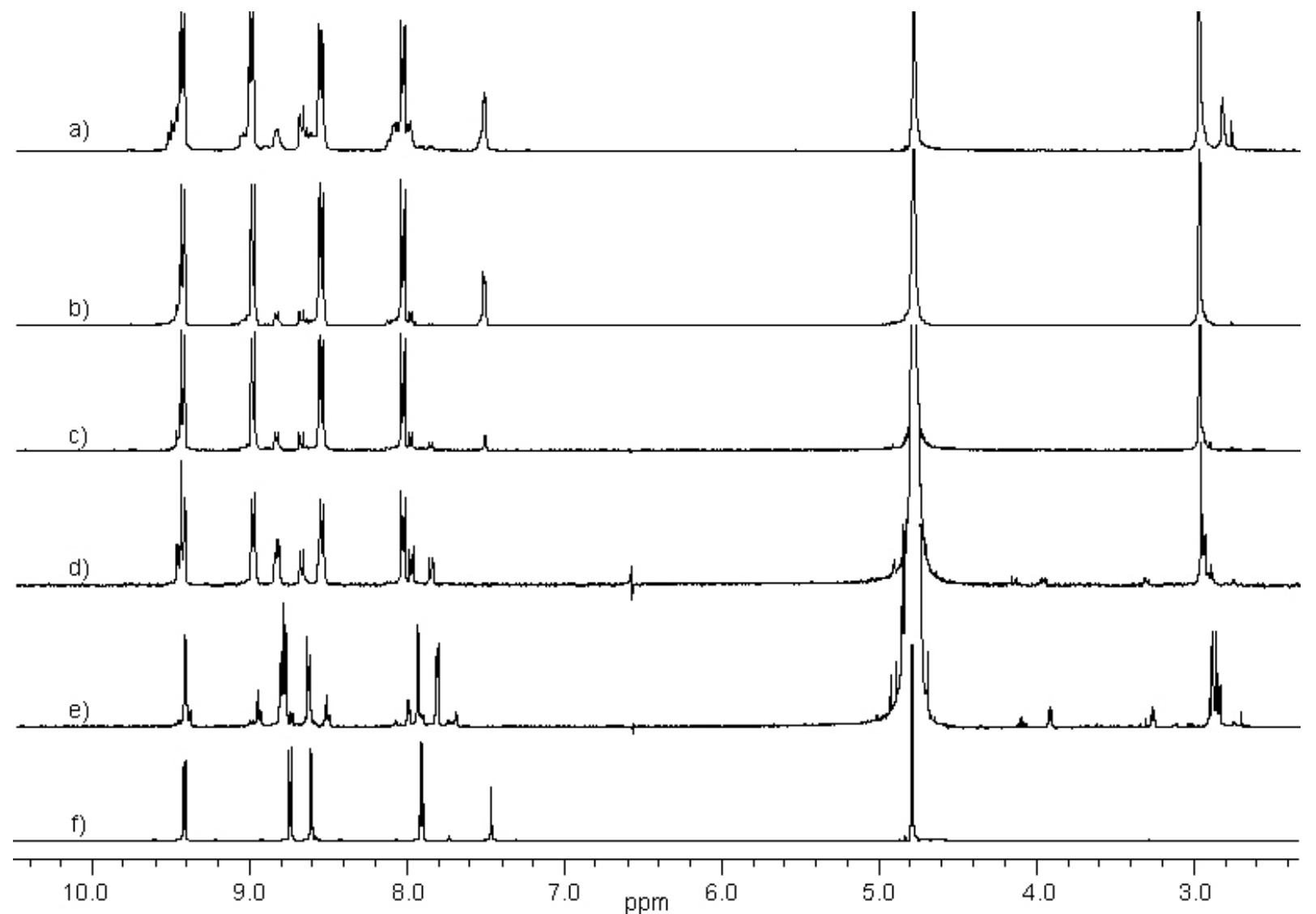

Figure S7 Partial ${ }^{1} \mathrm{H}$ NMR (300 MHz, $\left.\mathrm{D}_{2} \mathrm{O}\right)$ spectra of 3.8NO $\mathrm{N}_{3}$ a) $20 \mathrm{mM}$, b) $10 \mathrm{mM}$, c) $5 \mathrm{mM}$, d) 2 $\mathrm{mM}$, e) $0.5 \mathrm{mM}$, and f) $1.2 \mathrm{NO}_{3}$. 


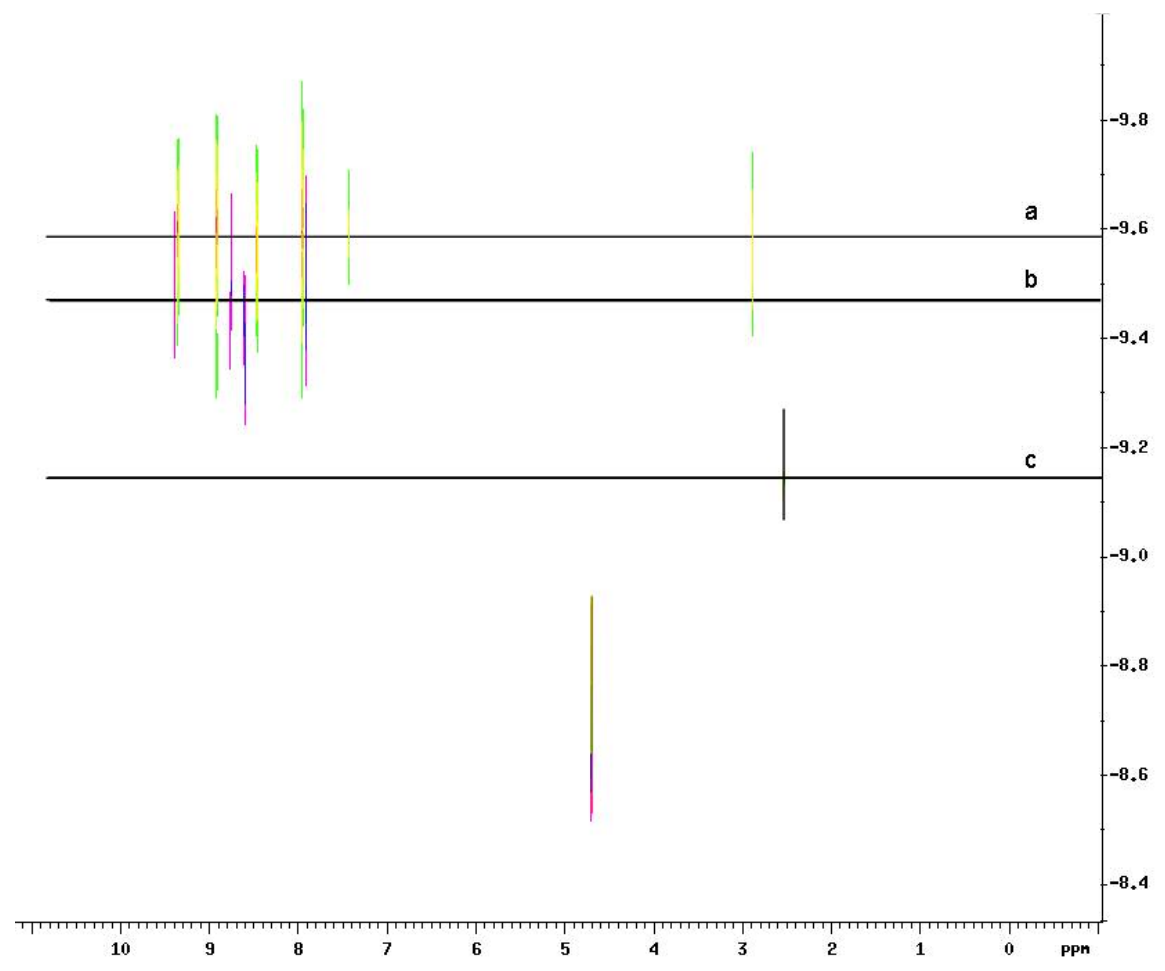

Figure S8 Superposed DOSY $\left(\mathrm{D}_{2} \mathrm{O}, 500 \mathrm{MHz}\right)$ experiments of a) metallocycle $3 \cdot 8 \mathrm{NO}_{3}$, b) ligand $\mathbf{1} \cdot 2 \mathrm{NO}_{3}$ and c) palladium complex 2.

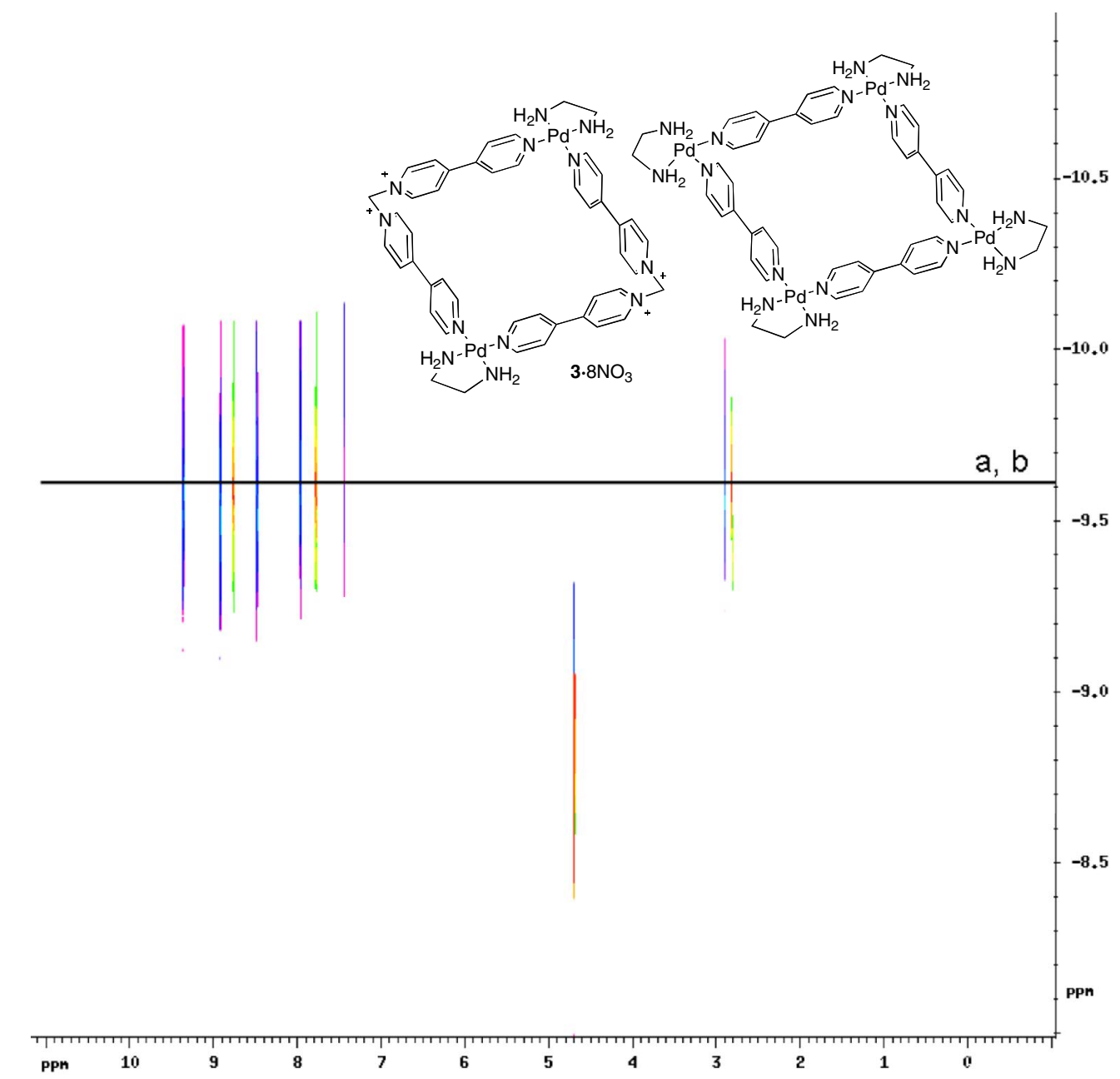

Figure S9 Superposed DOSY $\left(\mathrm{D}_{2} \mathrm{O}, 500 \mathrm{MHz}\right)$ experiments of a) metallocycle 3.8 $\mathrm{NO}_{3}$, b) Fujita's metallocycle. 

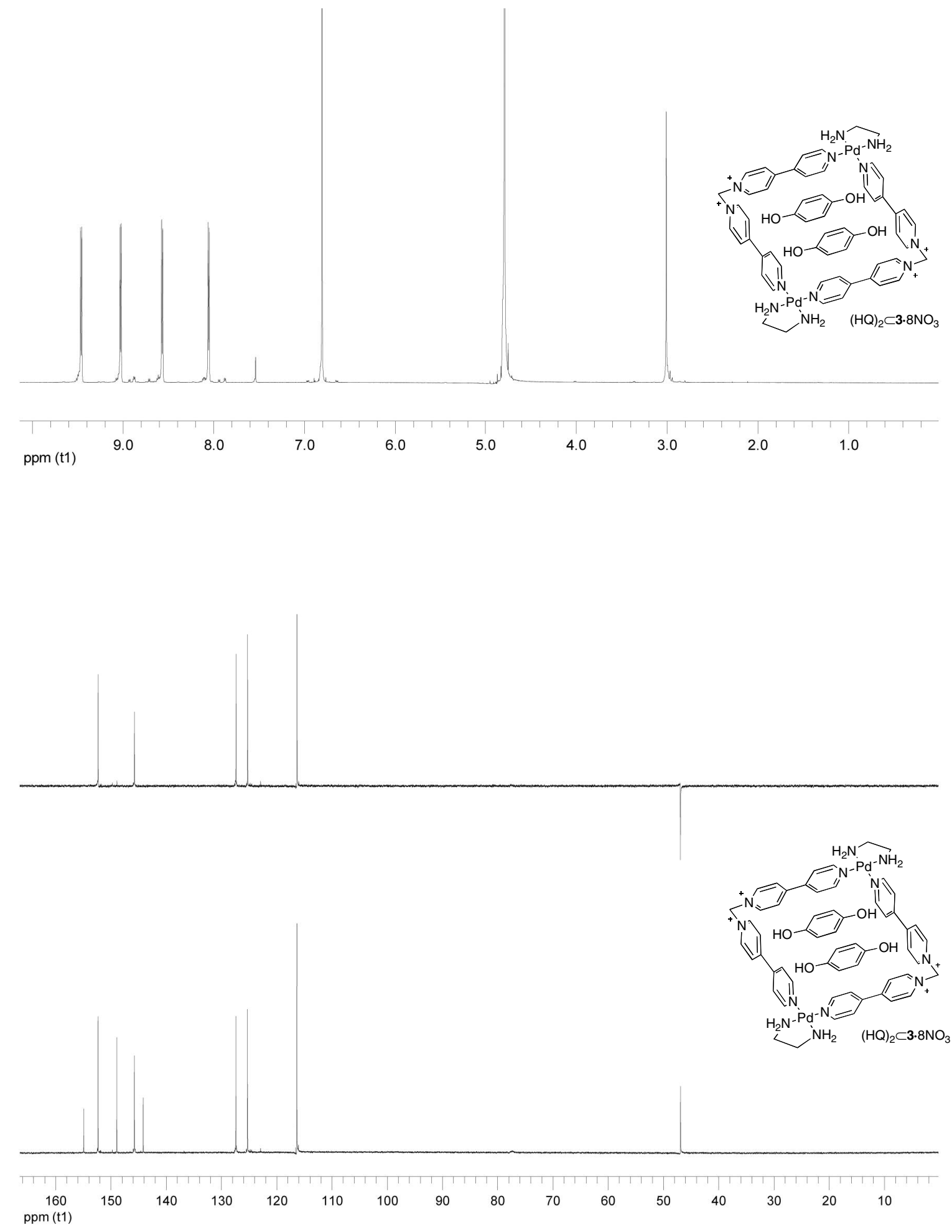

Figure S110 ${ }^{1} \mathrm{H}$ NMR (500 MHz, $\left.\mathrm{D}_{2} \mathrm{O}\right)$ (top) and ${ }^{13} \mathrm{C}$ NMR $\left(125 \mathrm{MHz}, \mathrm{D}_{2} \mathrm{O}\right.$ ) (bottom) spectra of $(\mathrm{HQ})_{2} \subset \mathbf{3} \cdot 8 \mathrm{NO}_{3}$. 

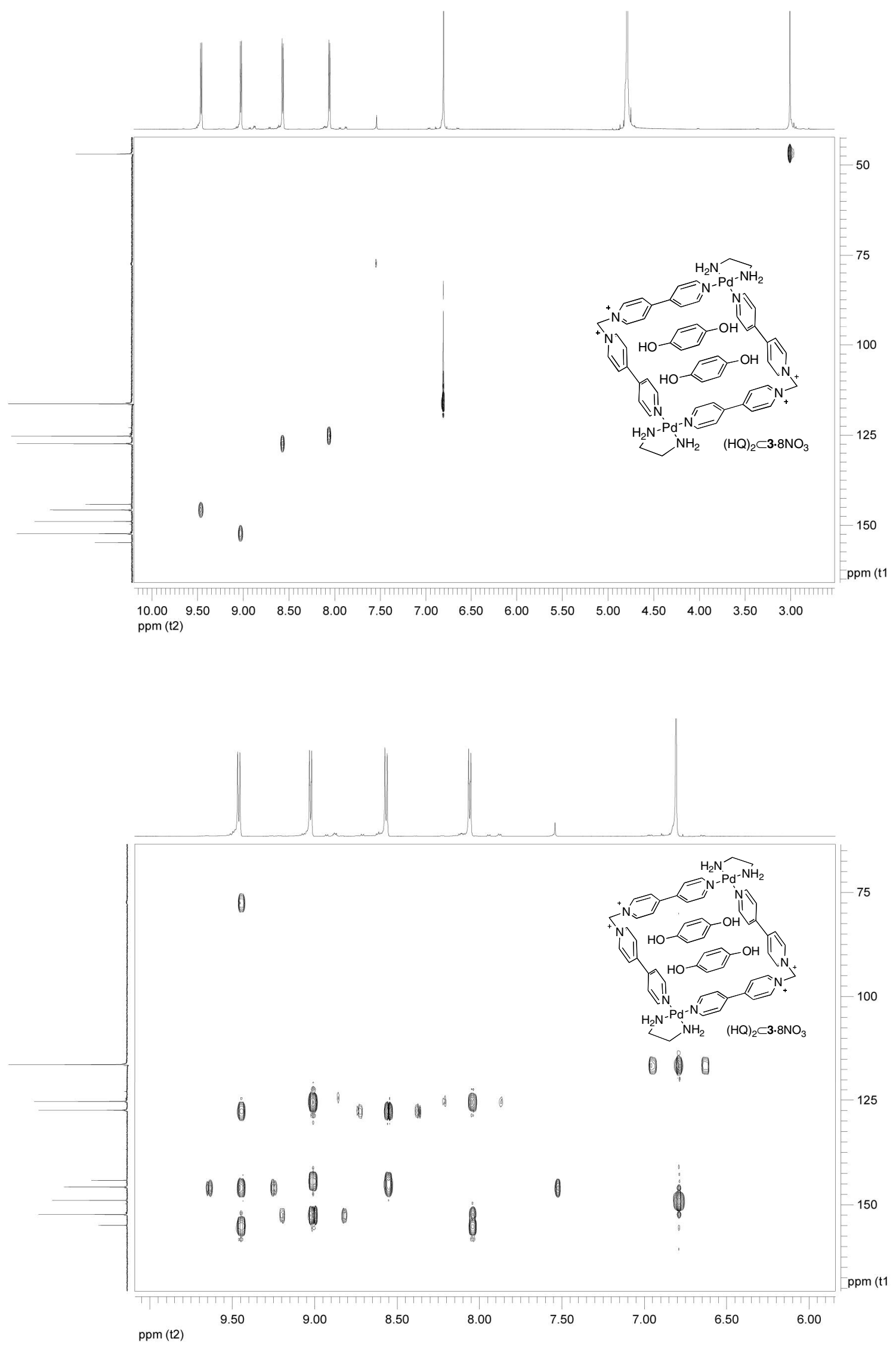

Figure S11 HSQC (500 MHz, $\mathrm{D}_{2} \mathrm{O}$ ) (top) and HMBC (500 MHz, $\mathrm{D}_{2} \mathrm{O}$ ) (bottom) spectra of $(\mathrm{HQ})_{2} \subset \mathbf{3} \cdot 8 \mathrm{NO}_{3}$. 


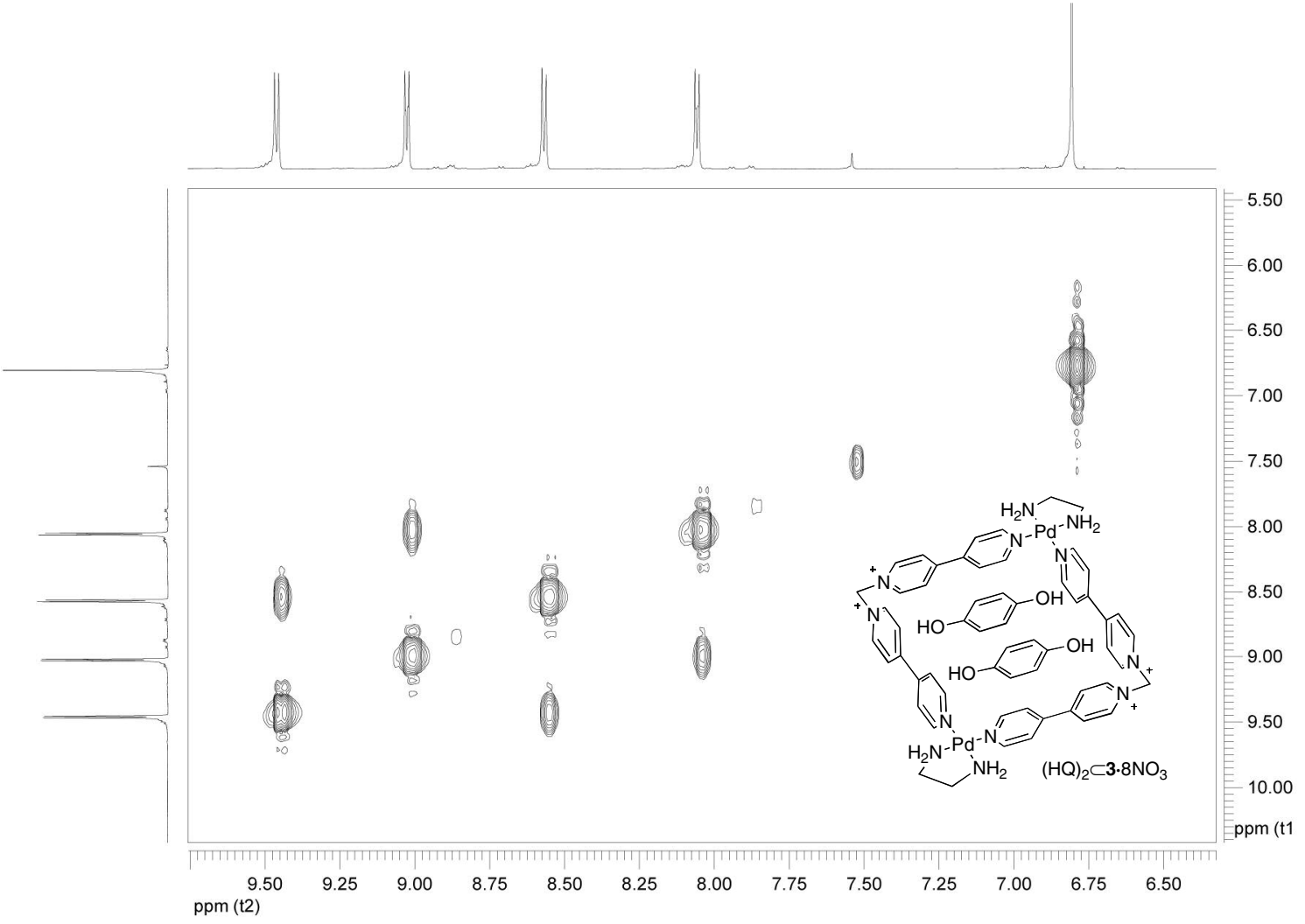

Figure S12 COSY $\left(500 \mathrm{MHz}, \mathrm{D}_{2} \mathrm{O}\right)$ spectrum of $(\mathrm{HQ})_{2} \subset \mathbf{3} \cdot 8 \mathrm{NO}_{3}$.

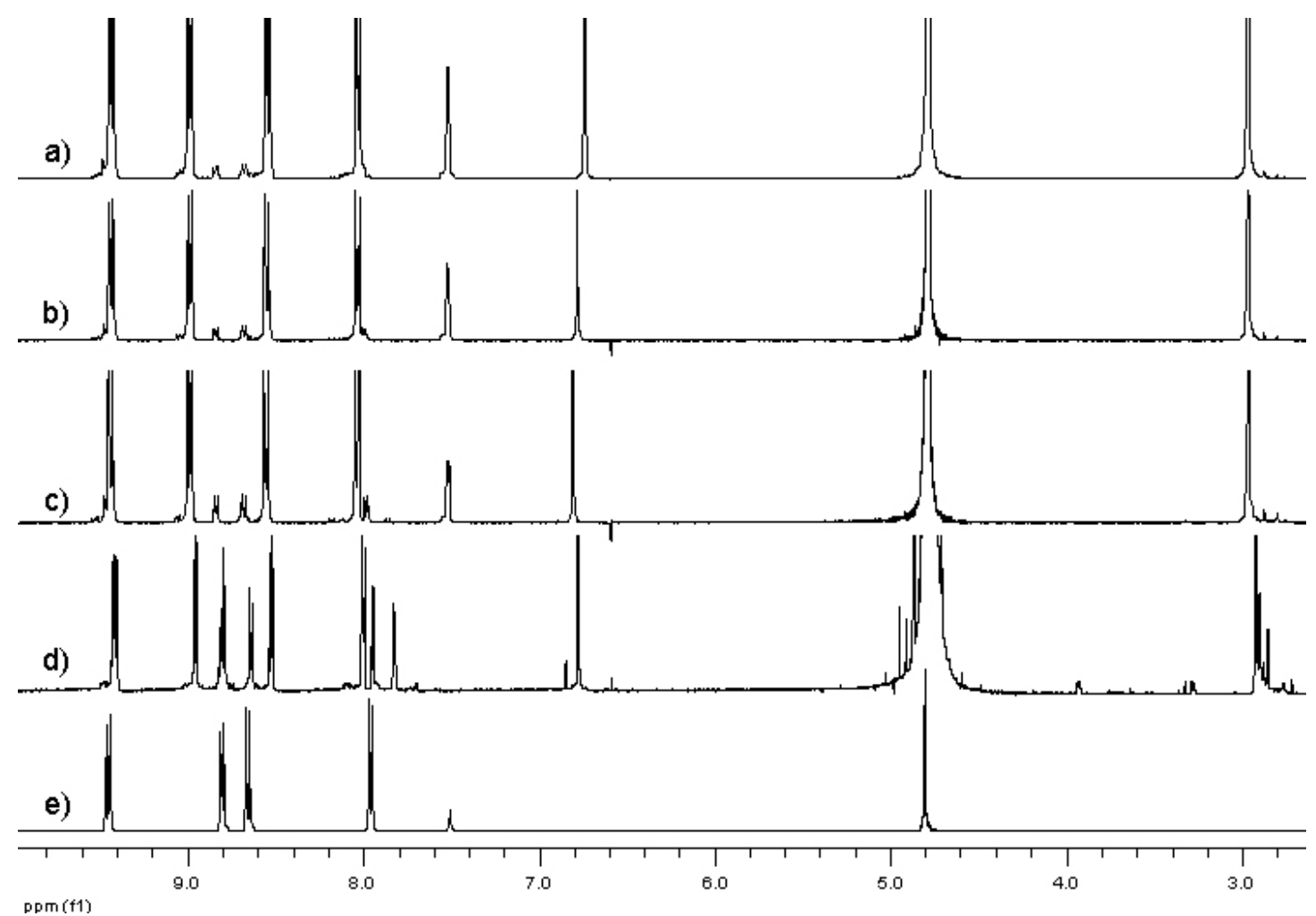

Figure S13 Partial ${ }^{1} \mathrm{H}$ NMR (300 MHz, $\left.\mathrm{D}_{2} \mathrm{O}\right)$ spectra of $(\mathrm{HQ})_{2} \subset \mathbf{3} \cdot 8 \mathrm{NO}_{3}$ a) $10 \mathrm{mM}$, b) $5 \mathrm{mM}$, c) 2 $\mathrm{mM}$, d) $0.5 \mathrm{mM}$, and e) $1.2 \mathrm{NO}_{3}$ 

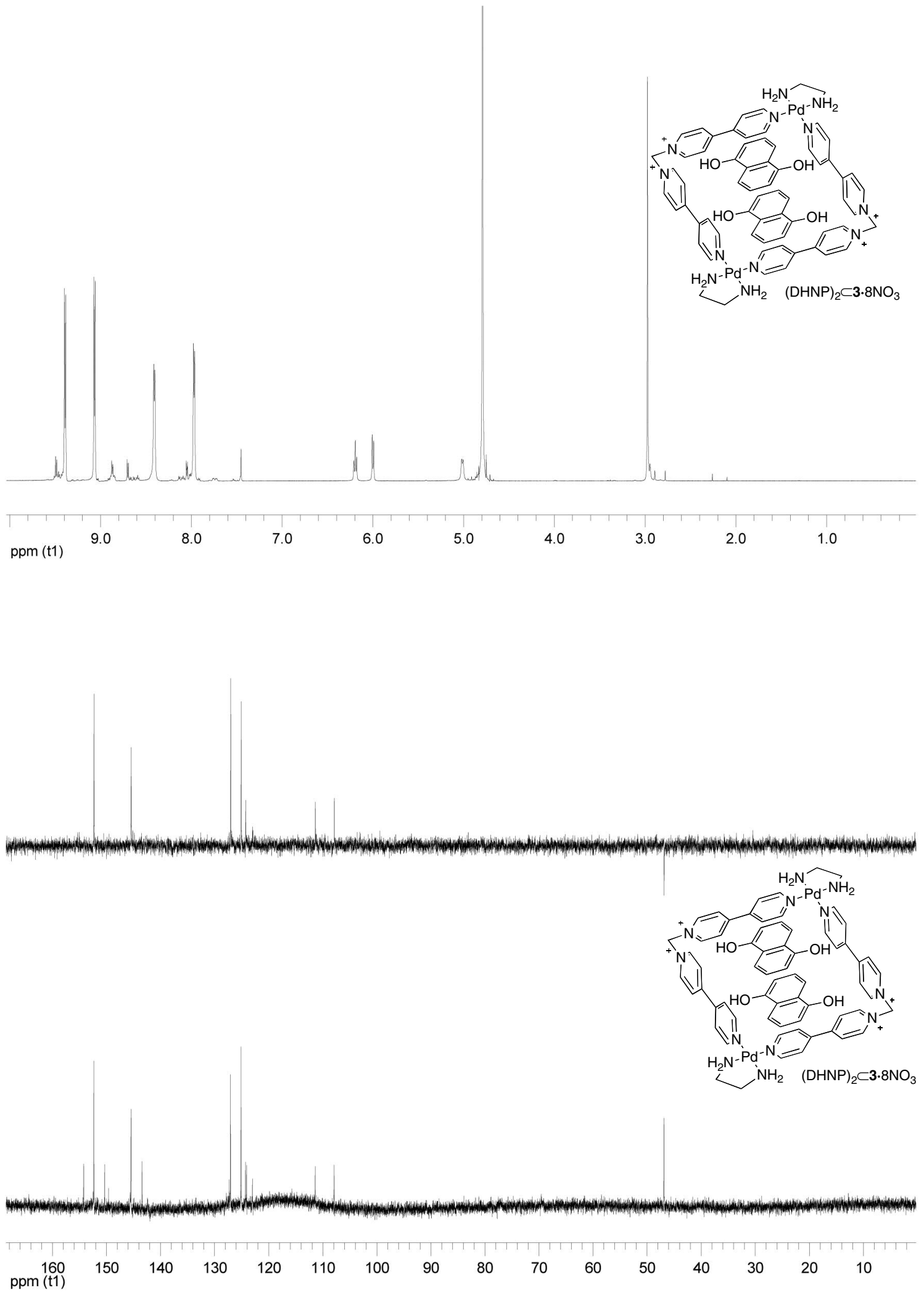

Figure S14 ${ }^{1} \mathrm{H}$ NMR $\left(500 \mathrm{MHz}, \mathrm{D}_{2} \mathrm{O}\right)$ (top) and ${ }^{13} \mathrm{C}$ NMR $\left(125 \mathrm{MHz}, \mathrm{D}_{2} \mathrm{O}\right)$ (bottom) spectra of $(\mathrm{DHNP})_{2} \subset \mathbf{3} \cdot 8 \mathrm{NO}_{3}$. 

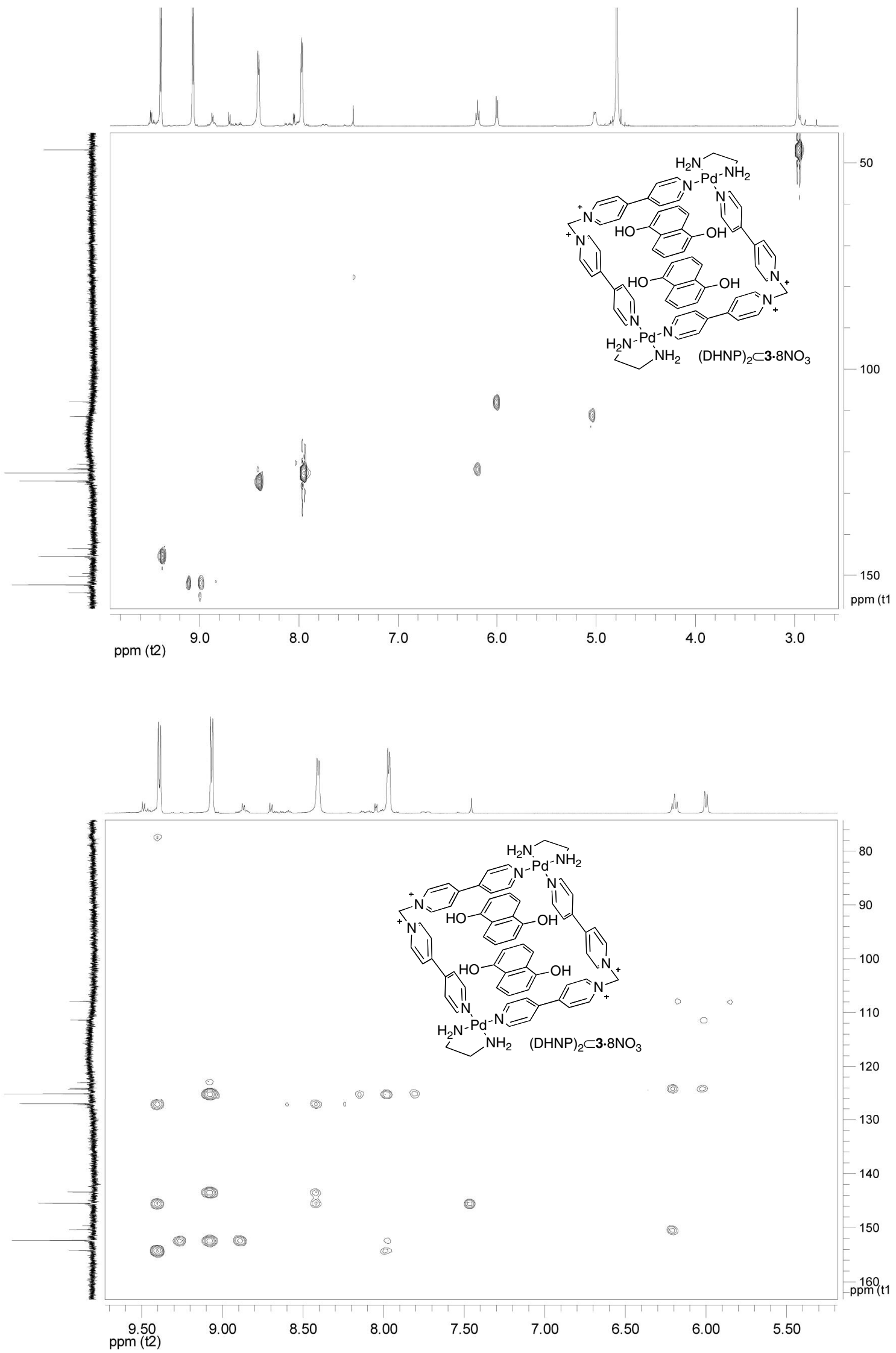

Figure S15 HSQC (500 MHz, $\mathrm{D}_{2} \mathrm{O}$ ) (top) and HMBC (500 MHz, $\mathrm{D}_{2} \mathrm{O}$ ) (bottom) spectra of $(\text { DHNP })_{2} \subset \mathbf{3} \cdot 8 \mathrm{NO}_{3}$. 


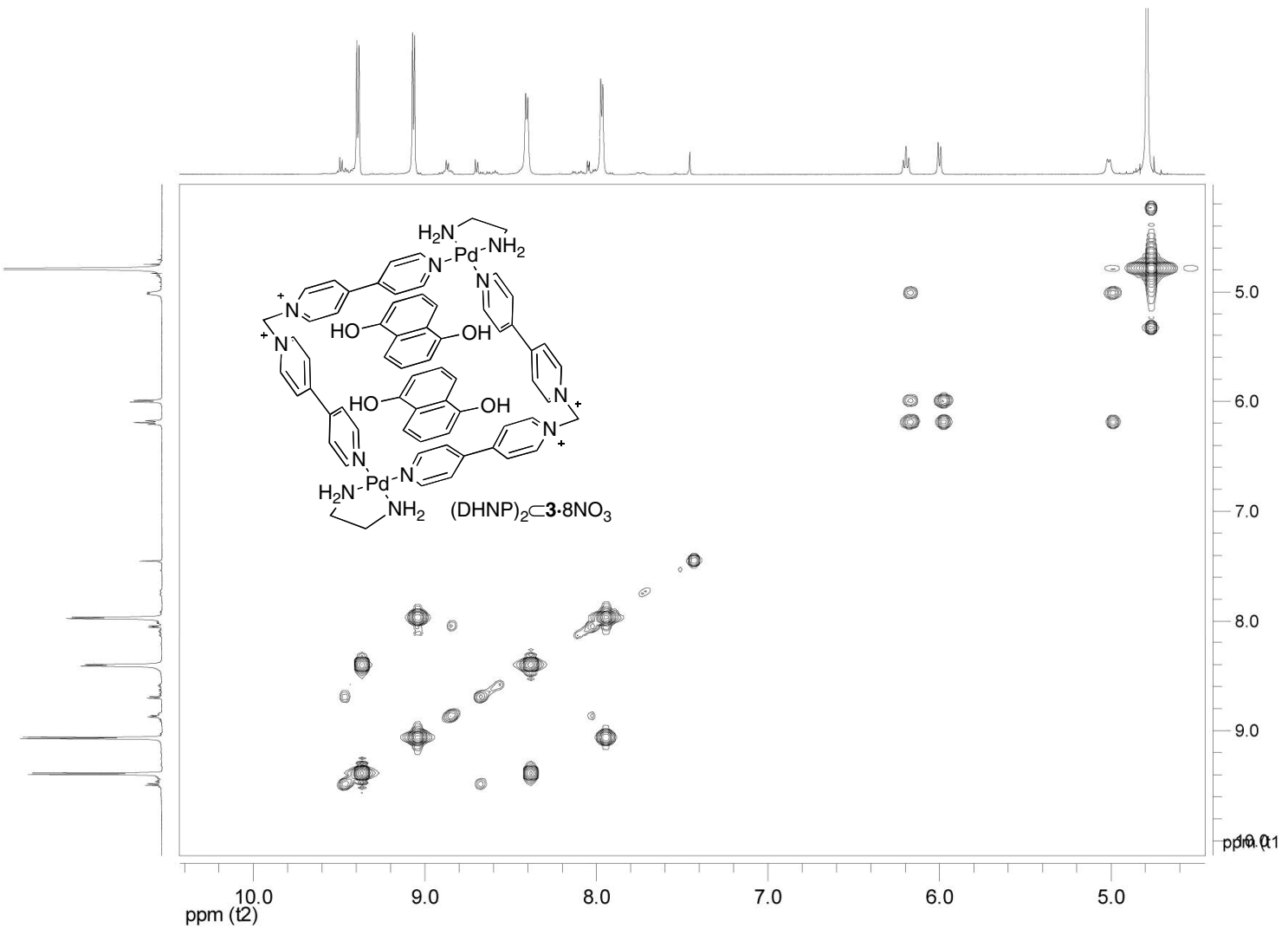

Figure S16 COSY $\left(500 \mathrm{MHz}, \mathrm{D}_{2} \mathrm{O}\right)$ spectrum of $(\mathrm{DHNP})_{2} \subset \mathbf{3} \cdot 8 \mathrm{NO}_{3}$.

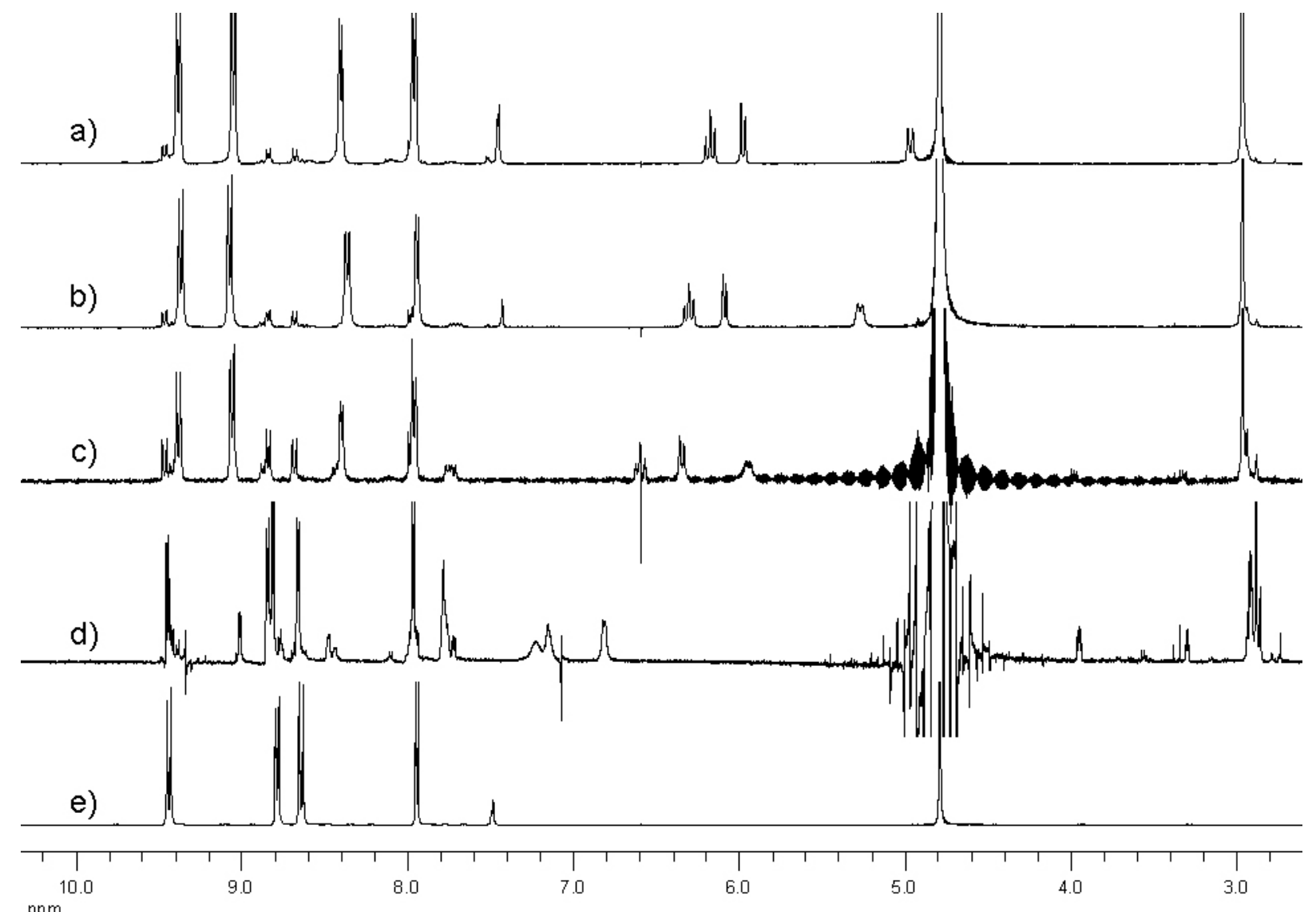

Figure S17 Partial ${ }^{1} \mathrm{H}$ NMR (300 MHz, $\left.\mathrm{D}_{2} \mathrm{O}\right)$ spectra of (DHNP) $)_{2} \subset \mathbf{3} \cdot 8 \mathrm{NO}_{3}$ a) $10 \mathrm{mM}$, b) $5 \mathrm{mM}$, c) $2 \mathrm{mM}$, d) $0.5 \mathrm{mM}$, and e) $1 \cdot 2 \mathrm{NO}_{3}$ 
a)

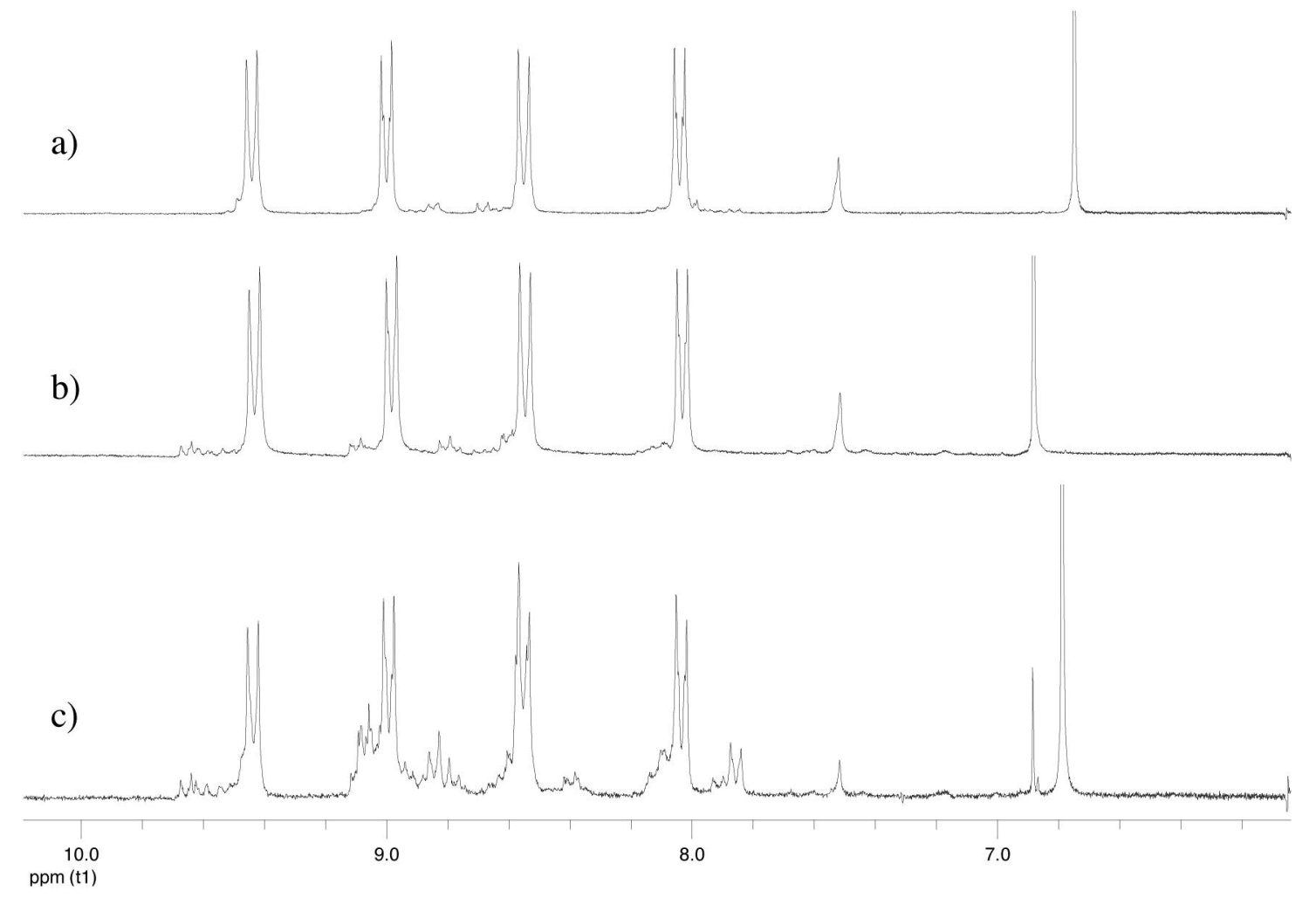

Figure S18 Partial ${ }^{1} \mathrm{H}$ NMR (200 MHz, $\left.\mathrm{D}_{2} \mathrm{O}, 298 \mathrm{~K}\right)$ spectra of: a) solution of ligand $\mathbf{1} \cdot 2 \mathrm{NO}_{3}(10$ $\mathrm{mM})$, Pd complex $2(10 \mathrm{mM})$ and HQ $(10 \mathrm{mM})$. b) solution a) +1.5 eq of CAN. c) solution b) +0.8 eq of $\mathrm{NaBH}_{4}$.

a)
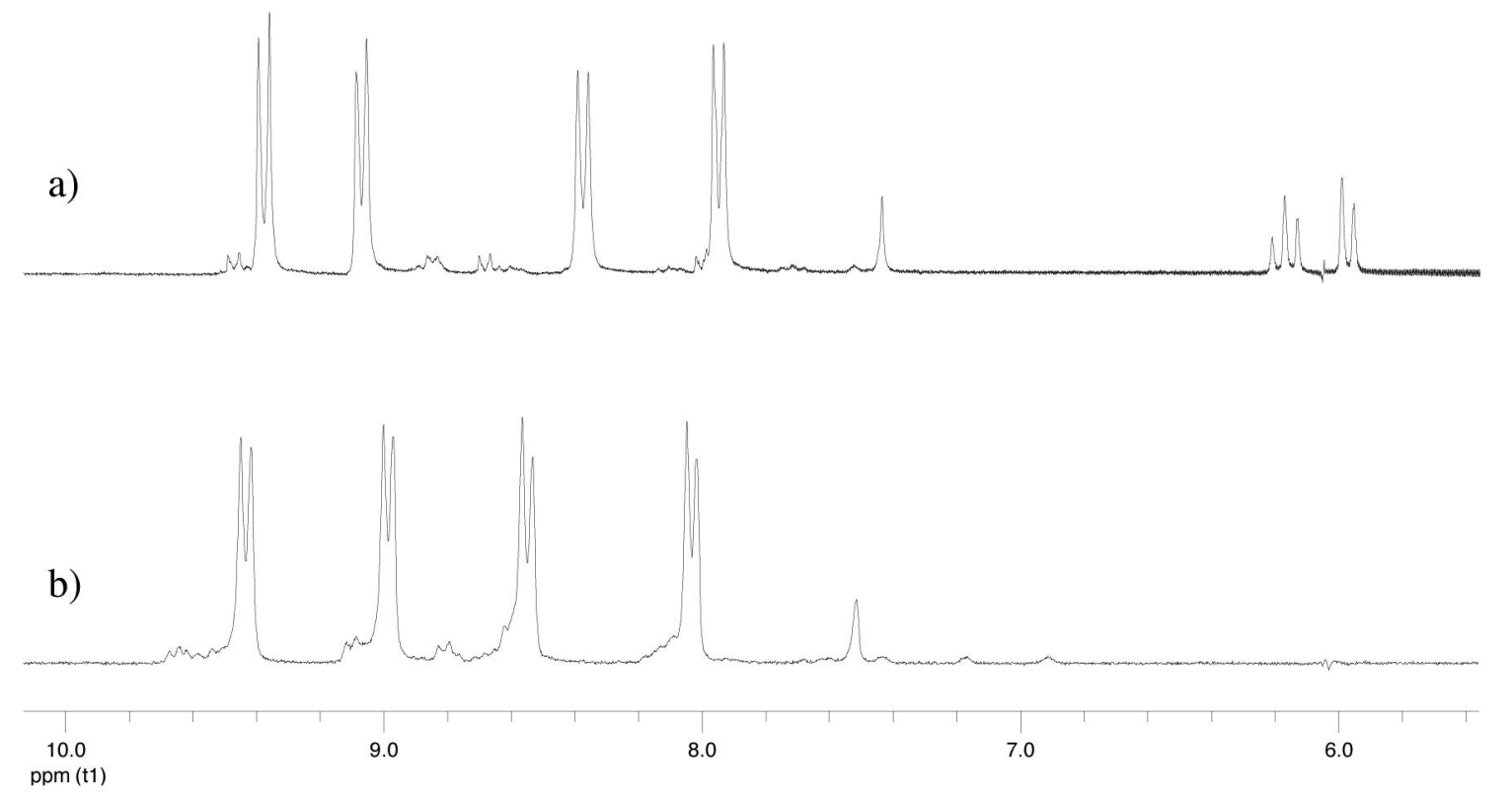

Figure S19 Partial ${ }^{1} \mathrm{H}$ NMR (200 MHz, $\mathrm{D}_{2} \mathrm{O}, 298 \mathrm{~K}$ ) spectra of: a) solution of ligand $\mathbf{1} \cdot 2 \mathrm{NO}_{3}$ $(10 \mathrm{mM})$, Pd complex $2(10 \mathrm{mM})$ and DHNP $(10 \mathrm{mM})$. b) solution a) + 1.5 eq of CAN. 


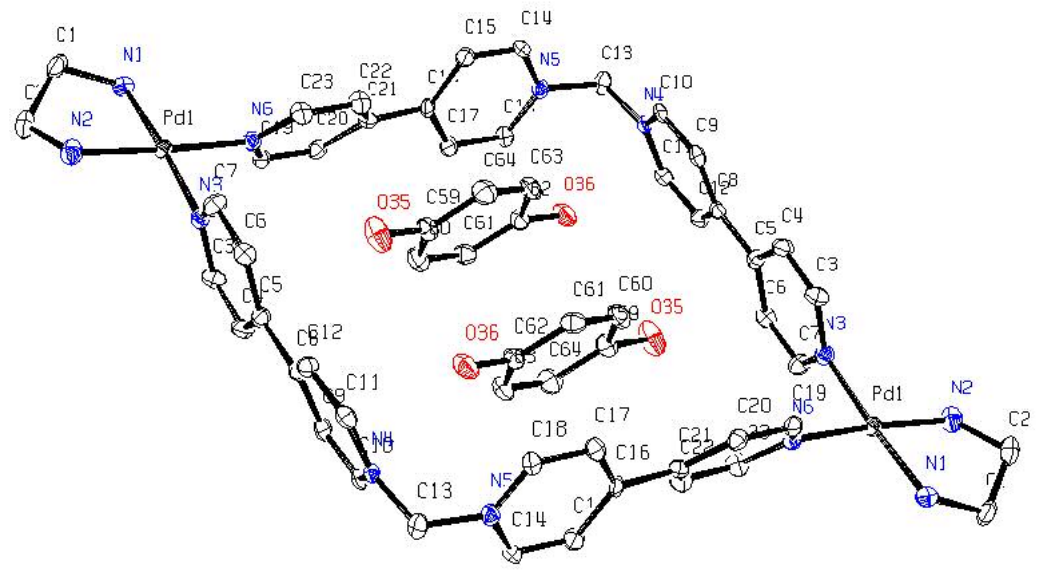

Figure S20 Single crystal X-ray Structure of (HQ) $)_{2} \subset \mathbf{3} \cdot 8 \mathrm{NO}_{3}$.

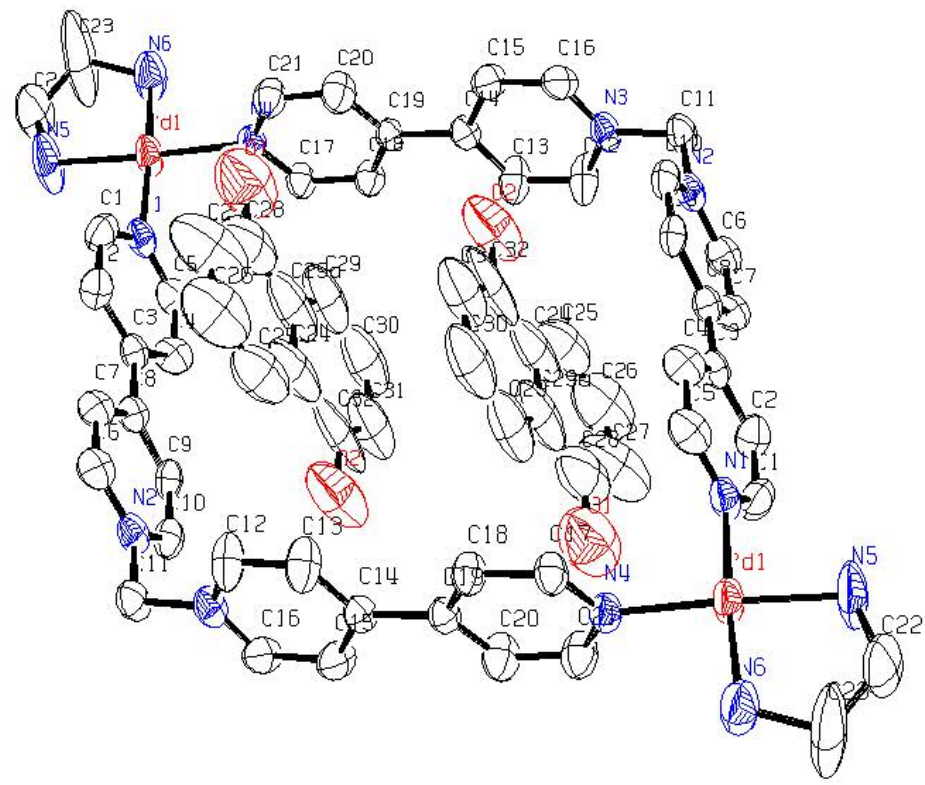

Figure S21 Single crystal X-ray Structure of (DHNP) $)_{2} \subset \mathbf{3} \cdot 8 \mathrm{NO}_{3}$. 